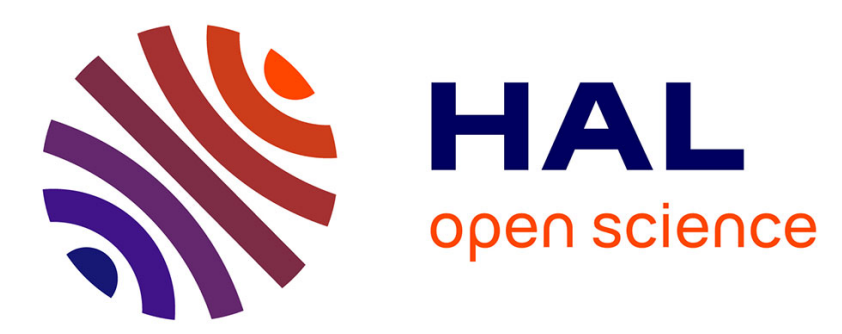

\title{
Pull-In Retarding in Nonlinear Nanoelectromechanical Resonators Under Superharmonic Excitation
}

\author{
Najib Kacem, Sébastien Baguet, Sebastien Hentz, Régis Dufour
}

\section{To cite this version:}

Najib Kacem, Sébastien Baguet, Sebastien Hentz, Régis Dufour. Pull-In Retarding in Nonlinear Nanoelectromechanical Resonators Under Superharmonic Excitation. Journal of Computational and Nonlinear Dynamics, 2012, 7 (2), pp.021011. 10.1115/1.4005435 . hal-00726024

\section{HAL Id: hal-00726024 https://hal.science/hal-00726024}

Submitted on 22 Sep 2014

HAL is a multi-disciplinary open access archive for the deposit and dissemination of scientific research documents, whether they are published or not. The documents may come from teaching and research institutions in France or abroad, or from public or private research centers.
L'archive ouverte pluridisciplinaire HAL, est destinée au dépôt et à la diffusion de documents scientifiques de niveau recherche, publiés ou non, émanant des établissements d'enseignement et de recherche français ou étrangers, des laboratoires publics ou privés. 


\title{
Pull-In Retarding in Nonlinear Nanoelectromechanical Resonators Under Superharmonic Excitation
}

\author{
Najib Kacem* \\ Université de Lyon \\ CNRS INSA-Lyon \\ LaMCoS UMR5259 \\ F-69621 Villeurbanne France \\ Email: najib.kacem@femto-st.fr \\ Sébastien Baguet \\ Université de Lyon \\ CNRS INSA-Lyon \\ LaMCoS UMR5259 \\ F-69621 Villeurbanne France \\ Email: sebastien.baguet@insa-lyon.fr \\ Sébastien Hentz \\ CEA/LETI - MINATEC, \\ 17 rue des Martyrs \\ F-38054 Grenoble, France \\ Email: sebastien.hentz@cea.fr \\ Régis Dufour \\ Université de Lyon \\ CNRS INSA-Lyon \\ LaMCoS UMR5259 \\ F-69621 Villeurbanne France \\ Email: regis.dufour@insa-lyon.fr
}

In order to compensate for the loss of performance when scaling resonant sensors down to NEMS, a complete analytical model including all main sources of non linearities is presented as a predictive tool for the dynamic behavior of clamped-clamped nanoresonators electrostatically actuated. The nonlinear dynamics of such NEMS under superharmonic resonance of order half their fundamental natural frequencies is investigated. It is shown that the critical amplitude has the same dependence on the quality factor $Q$ and the thickness $h$ as the case of the primary resonance. Finally, a way to retard the pull-in by decreasing the AC voltage is proposed in order to enhance the performance of NEMS resonators.

\section{Introduction}

The small size of NEMS resonators combined with their physical attributes make them quite attractive and suitable for a wide range of technological applications such as ultrasensitive force and mass sensing, narrow band filtering, and time keeping. However, at this size regime, nonlinearities occur sooner which reduces the dynamic range of such devices.

It is a challenge to achieve large-amplitude motion of NEMS resonators without deteriorating their frequency stability [1]. The relative frequency noise spectral density [2] of a NEMS resonator is given by:

$$
S_{f}=\left(\frac{1}{2 Q}\right)^{2} \frac{S_{x}}{P_{0}}
$$


where $S_{x}$ is the displacement spectral density and $P_{0}$ is the displacement carrier power, ie the RMS drive amplitude of the resonator $\frac{1}{2} A^{2}$. Remarkably, driving the resonator at large oscillation amplitude leads to better signal to noise ratio (SNR) and, thus, simplifies the design of the electronic feedback loop. However, doing so in the nonlinear regime reduces the sensor performances since its frequency stability becomes dependent on its oscillation amplitude. Moreover, even when NEMS resonators are used as oscillators in closed-loop, a large part of noise mixing $[3,4]$ due to nonlinearities drastically reduces their dynamic range and alters their detection limit.

A nonlinear model for a clamped-clamped microbeam was introduced in a previous work [5] and the ability to suppress the hysteresis of the nonlinear frequency response under primary resonance was demonstrated by third order nonlinearity cancellation. Thus the performances of resonant sensors might be enhanced by driving the resonator beyond its critical amplitude [6-8].

Nevertheless, the operating domain of the third order nonlinearity cancellation is limited by the occurrence of the mixed behavior [7-10] which appears proportionally sooner with respect to the resonator size and closer to the critical amplitude. Moreover, at this oscillation level and in the case of electrostatic forces actuation, pull-in (the upper bound limit) is easily reachable which makes the electrical characterization of NEMS resonators quite difficult and limits the possible domain of resolution enhancement.

In order to avoid such complications, we investigate the dynamics of NEMS resonators under superharmonic resonance. Jin and Wang [11] showed that driving a microbeam of a resonant microsensor by a superharmonic excitation of order one-half increases the signal-to-crosstalk ratio as compared to driving it at primary resonance.

The dynamic behavior of MEMS resonators under secondary resonances has been investigated by many authors. Turner et al [12] studied the response of a comb-drive device to a parametric excitation that offers interesting behavior, and a possibility for novel applications such as parametric amplification [13-15] and noise squeezing [13]. Younis and Nayfeh [16] and Abdel-Rahman and Nayfeh [17] used the method of multiple scales to study the response of an electrostatically deflected microbeam based resonator to a primaryresonance excitation, a superharmonic-resonance excitation of order two, and a subharmonic-resonance excitation of order one-half. Since they are based on perturbation methods, these models yield accurate results only for small AC amplitudes and hence small motions.

Younis et al [18] and Nayfeh and Younis [19] studied the global dynamics of MEMS resonators under superharmonic excitation and showed that the dynamic pull-in phenomenon can occur for a superharmonic excitation at an electric load much lower than that predicted by a static analysis.

In this paper, the dynamics of a nanoelectromechanical resonator, actuated around half its fundamental natural frequency, is simulated. A complete analytical model incorporating all main sources of nonlinearities is used based on the Galerkin discretisation coupled with multiple scales pertur- bation technique.

The dependences of the resonator critical amplitude on the physical parameters are analyzed in order to compare it with the primary resonance case. The results are shown with respect to the quality factor and actuation voltages. Finally, the dynamic behavior of the resonator up to the pull-in instability $[19,20]$ is tracked and a solution to enhance the resonator performances is provided.

\section{Model}

Existing models [16-20] for the nonlinear dynamics of MEMS resonators with relatively high capacitive variations concern designs with only one electrode for both actuation and sensing. NEMS resonators though, have low capacitive variations, and it is almost necessary to use a two port measurement, i.e. to separate detection and actuation electrodes in order to enhance the signal to background ratio. Moreover, the use of different gaps $\left(g_{d}<g_{a}\right)$ enables the maximization of the detection signal.

The resonator considered here is presented in Fig. 1. It consists of a clamped-clamped microbeam actuated by an electric load $v(\tilde{t})=-V_{d c}+V_{a c} \cos (\tilde{\Omega} \tilde{t})$, where $V_{d c}$ is the $D C$ polarization voltage, $V_{a c}$ is the amplitude of the applied $A C$ voltage, and $\tilde{\Omega}$ is the forcing frequency.

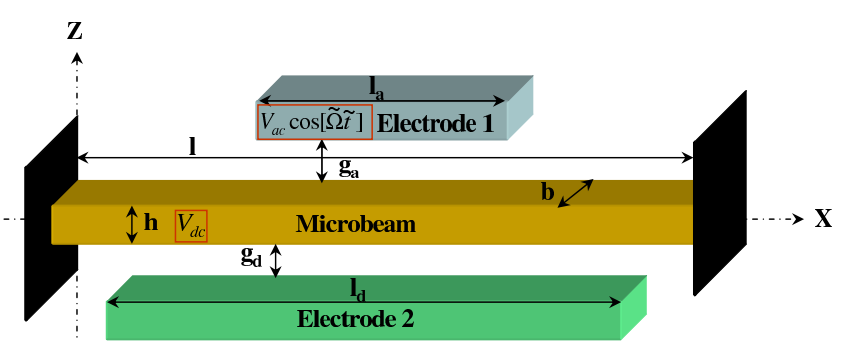

Fig. 1. Schema of an electrically actuated microbeam

\subsection{Equation of motion}

The transverse deflection of the microbeam $w(x, t)$ is governed by the nonlinear Euler-Bernoulli equation, which is the commonly used approximate equation of motion for a thin beam [21]

$$
\begin{array}{r}
E I \frac{\partial^{4} \tilde{w}(\tilde{x}, \tilde{t})}{\partial \tilde{x}^{4}}+\rho b h \frac{\partial^{2} \tilde{w}(\tilde{x}, \tilde{t})}{\partial \tilde{t}^{2}}+\tilde{c} \frac{\partial \tilde{w}(\tilde{x}, \tilde{t})}{\partial \tilde{t}} \\
-\left[\tilde{N}+\frac{E b h}{2 l} \int_{0}^{l}\left[\frac{\partial \tilde{w}(\tilde{x}, \tilde{t})}{\partial \tilde{x}}\right]^{2} d \tilde{x}\right] \frac{\partial^{2} \tilde{w}(\tilde{x}, \tilde{t})}{\partial \tilde{x}^{2}} \\
=\frac{1}{2} \varepsilon_{0} \frac{b C_{n 1}\left[V_{a c} \cos (\tilde{\Omega} \tilde{t})-V_{d c}\right]^{2}}{\left(g_{a}-\tilde{w}(\tilde{x}, \tilde{t})\right)^{2}} H_{1}(\tilde{x}) \\
-\frac{1}{2} \varepsilon_{0} \frac{b C_{n 2} V_{d c}^{2}}{\left(g_{d}+\tilde{w}(\tilde{x}, \tilde{t})\right)^{2}} H_{2}(\tilde{x})
\end{array}
$$




$$
\begin{aligned}
& H_{1}(\tilde{x})=H\left(\tilde{x}-\frac{l+l_{a}}{2}\right)-H\left(\tilde{x}-\frac{l-l_{a}}{2}\right) \\
& H_{2}(\tilde{x})=H\left(\tilde{x}-\frac{l+l_{d}}{2}\right)-H\left(\tilde{x}-\frac{l-l_{d}}{2}\right)
\end{aligned}
$$

where $\tilde{x}$ is the position along the microbeam length, $\tilde{c}$ is the linear viscous damping per unit length, $E$ and $I$ are the Young's modulus and moment of inertia of the cross section. $\tilde{N}$ is the applied tensile axial force due to the residual stress on the silicon, $\tilde{t}$ is time, $\rho$ is the material density, $h$ is the microbeam thickness, $g_{a}$ and $g_{d}$ are respectively the actuation and the sensing capacitor gap thickness, $\varepsilon_{0}$ is the dielectric constant of the gap medium. The last term in Eq. (2) represents an approximation of the electric force assuming a resonator design with 2 stationary electrodes : the first one is devoted to actuation while the second one is used for sensing. The fringing field effect [22] was taken into account using the coefficients $C_{n i}$. Since the electrodes do not act on the whole length of the beam, the electrostatic force distributions are modeled by means of Heaviside functions $H(\tilde{x})$. The microbeam is subject to the following boundary conditions

$$
\tilde{w}(0, \tilde{t})=\tilde{w}(l, \tilde{t})=\frac{\partial \tilde{w}}{\partial \tilde{x}}(0, \tilde{t})=\frac{\partial \tilde{w}}{\partial \tilde{x}}(l, \tilde{t})=0
$$

\subsection{Normalization}

For convenience and equations simplicity, the following nondimensional variables are introduced

$$
w=\frac{\tilde{w}}{g_{d}}, x=\frac{\tilde{x}}{l}, t=\frac{\tilde{t}}{\tau}
$$

where $\tau=\frac{2 l^{2}}{h} \sqrt{\frac{3 \rho}{E}}$.

Substituting Eq. (6) into Eqns. (2) and (5) yields

$$
\begin{array}{r}
\frac{\partial^{4} w}{\partial x^{4}}+\frac{\partial^{2} w}{\partial t^{2}}+c \frac{\partial w}{\partial t}+\alpha_{2} C_{n 2} \frac{V_{d c}^{2}}{(1+w)^{2}} H_{2}(x) \\
=\alpha_{2} C_{n 1} \frac{\left[V_{a c} \cos (\Omega t)-V_{d c}\right]^{2}}{\left(R_{g}-w\right)^{2}} H_{1}(x) \\
+\left[N+\alpha_{1} \int_{0}^{1}\left[\frac{\partial w}{\partial x}\right]^{2} d x\right] \frac{\partial^{2} w}{\partial x^{2}} \\
w(0, t)=w(1, t)=\frac{\partial w}{\partial x}(0, t)=\frac{\partial w}{\partial x}(1, t)=0
\end{array}
$$

The parameters appearing in Eq. (7) are

$$
\begin{aligned}
& H_{2}(x)=H\left(x-\frac{l+l_{a}}{2 l}\right)-H\left(x-\frac{l-l_{a}}{2 l}\right) \\
& H_{2}(x)=H\left(x-\frac{l+l_{d}}{2 l}\right)-H\left(x-\frac{l-l_{d}}{2 l}\right)
\end{aligned}
$$

$$
\begin{gathered}
c=\frac{\tilde{c} l^{4}}{E I \tau}, N=\frac{\tilde{N} l^{2}}{E I}, \alpha_{1}=6\left[\frac{g_{a}}{h}\right]^{2} \\
R_{g}=\frac{g_{a}}{g_{d}}, \alpha_{2}=6 \frac{\varepsilon_{0} l^{4}}{E h^{3} g_{a}^{3}}, \Omega=\tilde{\Omega} \tau
\end{gathered}
$$

\subsection{Solving}

The beam total displacement $w(x, t)$ can be written as a sum of a static $d c$ displacement $w_{s}(x)$ and a time-varying ac displacement $w_{d}(x, t)$. However, in the present case, the static deflection can be considered as negligible because the measured quality factors $Q$ are in the range of $2.10^{3}-10^{4}$ and $V d c \leq 40 V a c$. Indeed, the ratio between the static and the dynamic deflections is

$$
\frac{w_{s}(x)}{w_{d}(x, t)} \approx \frac{V d c}{2 Q . V a c} \leq 1 \%
$$

A reduced-order model is generated by modal decomposition, transforming Eq. (7) into a finite-degree-of-freedom system consisting in ordinary differential equations in time. The undamped linear mode shapes of the straight microbeam are used as basis functions in the Galerkin procedure, and the deflection is approximated by

$$
w(x, t)=\sum_{k=1}^{n} a_{k}(t) \phi_{k}(x)
$$

where $n$ is the number of retained modes (size of the modal basis), $a_{k}(t)$ is the $k^{t h}$ generalized coordinate and $\phi_{k}(x)$ is the $k^{\text {th }}$ linear undamped mode shape of the straight microbeam. The linear undamped mode shapes $\phi_{k}(x)$ are governed by:

$$
\begin{array}{r}
\frac{d^{4} \phi_{k}(x)}{d x^{4}}=\lambda_{k}^{2} \phi_{k}(x) \\
\phi_{k}(0)=\phi_{k}(1)=\phi_{k}^{\prime}(0)=\phi_{k}^{\prime}(1)
\end{array}
$$

The analytical form of the eigenmodes is given by

$$
\begin{array}{r}
\phi_{k}(x)=A_{k}\left\{\left[\frac{\cosh \lambda_{k}-\cos \lambda_{k}}{\sin \lambda_{k}-\sinh \lambda_{k}}\right]\left[\sin \lambda_{k} x-\sinh \lambda_{k} x\right]\right. \\
\left.+\cos \lambda_{k} x-\cosh \lambda_{k} x\right\}
\end{array}
$$

with $\lambda_{k}$ solutions of the transcendental equation

$$
1-\cos \lambda_{k} \cosh \lambda_{k}=0
$$


These functions are a modal basis for the scalar product

$$
\langle u, v\rangle=\int_{0}^{1} u(x) v(x) d x
$$

and the coefficients $A_{k}$ are chosen to normalize the eigenmodes such that $\left\langle\phi_{i}, \phi_{j}\right\rangle=\delta_{i j}$, where $\delta_{i j}$ is the Kronecker symbol.

Let Eq. (7) be multiplied by $\phi_{k}(x)\left[(1+w)\left(R_{g}-w\right)\right]^{2}$ in order to include the complete contribution of the nonlinear electrostatic forces in the resonator dynamics without approximation. This method, which has been used by Nayfeh et al. $[19,20]$ for a one-port resonator, has some disadvantages like the non orthogonality of the operator $w^{4} \frac{\partial^{4} w}{\partial x^{4}}$ with respect to the undamped linear mode shapes of the resonator, the increase of the nonlinearity level in the normalized equation of motion (7) as well as the incorporation of new nonlinear terms such as the Van der Pol damping. Nevertheless, the resulting equation contains less parametric terms than if the nonlinear electrostatic forces were expanded in Taylor series and the solution of nonlinear problem is valid for large displacements of the beam up to the sensing gap.

Substituting Eqns. (14) and (15) into the resulting equation, and then integrating the outcome from $x=0$ to $x=1$ yields a system of coupled ordinary differential equations in time.

For several nonlinear mechanical systems such as shells [23], many modes are needed in order to build the frequency response without losing any physical information concerning the nonlinear coupling between the modes. Nevertheless, in our case (simple beam resonators), the first mode can be considered as the dominant mode of the system and the higher modes can be neglected. This has been demonstrated numerically using time integration [7] as well as shooting and harmonic balance method coupled with an asymptotic numerical continuation technique [24]. Thus, only one mode is considered $(n=1)$.

Moreover, since $V_{a c}<<V_{d c}$ and consequently $V_{a c}^{2}<<$ $V_{a c} . V_{d c}$, the effects of the fast harmonics (proportional to $V_{a c}^{2}$ ) on the slow excitations (proportional to $V_{a c} . V_{d c}$ ) are negligible [25]. Thus, the linear and nonlinear terms related to the second harmonic has not been considered and the following equation is obtained:

$$
\begin{array}{r}
\ddot{a}_{1}+c \dot{a}_{1}+\omega_{n}{ }^{2} a_{1}+\mu_{1} a_{1} \ddot{a}_{1}+\mu_{2} a_{1}{ }^{2} \ddot{a}_{1}+\mu_{3} a_{1}{ }^{3} \ddot{a}_{1} \\
+\mu_{4} a_{1}{ }^{4} \ddot{a}_{1}+c \mu_{1} a_{1} \dot{a}_{1}+c \mu_{2} a_{1}{ }^{2} \dot{a}_{1}+c \mu_{3} a_{1}{ }^{3} \dot{a}_{1} \\
+c \mu_{4} a_{1}{ }^{4} \dot{a}_{1}+\chi_{2} a_{1}{ }^{2}+\chi_{3} a_{1}{ }^{3}+\chi_{4} a_{1}{ }^{4}+\chi_{5} a_{1}{ }^{5} \\
+\chi_{6} a_{1}{ }^{6}+\chi_{7} a_{1}{ }^{7}+v+\zeta_{0} \cos (\Omega t) \\
+\zeta_{1} a_{1} \cos (\Omega t)+\zeta_{2} a_{1}{ }^{2} \cos (\Omega t)=0
\end{array}
$$

Some canonical nonlinear terms can be identified in Eq. (20), such as the Duffing non linearity $\left(\chi_{3} a_{1}{ }^{3}\right)$, the nonlinear Van der Pol damping $\left(c \mu_{2} a_{1}^{2} \dot{a}_{1}\right)$ as well as the parametric excitations $\left(\zeta_{1} a_{1} \cos (\Omega t)+\zeta_{2} a_{1}{ }^{2} \cos (\Omega t)\right)$ which are due to the fact that the beam is actuated by two electrodes. Indeed, for one-port resonators, the parametric terms are eliminated by the pre-multiplication of the equation of motion by the denominator of the electrostatic force [24].

Other terms correspond to high-level nonlinearities and nonlinear parametric excitations. All these terms come from the coupling between the mechanical and the electrostatic nonlinearities as well as the nonlinear coupling between both electrostatic forces due to the pre-multiplication of Eq. (7) by the common denominator of both electrostatic forces in actuation and sensing. The appendix shows the expressions of all the integration parameters appearing in Eq. (20), which can be easily computed with any computational software.

To analyse this equation of motion, it proves convenient to invoke perturbation techniques which work well with the assumptions of "small" excitation and damping $(Q>10)$, typically valid in MEMS resonators. In this paper, the method of multiple scales [26] is used to attack Eq. (20) in order to determine a uniformly valid approximate solution. To this end, we seek a first-order uniform solution in the form

$$
a_{1}(t, \varepsilon)=a_{10}\left(T_{0}, T_{1}\right)+\varepsilon a_{11}\left(T_{0}, T_{1}\right)+\cdots
$$

where $\varepsilon$ is the small nondimensional bookkeeping parameter, $T_{0}=t$ and $T_{1}=\varepsilon t$. Since the non linear response to a superharmonic resonance excitation of order two is analyzed, the nearness of $\Omega$ to $\frac{\omega_{n}}{2}$ is expressed by introducing the detuning parameter $\sigma$ according to

$$
2 \Omega=\omega_{n}+\varepsilon \sigma
$$

Substituting Eq. (21) into Eq. (20) and equating coefficients of like powers of $\varepsilon$ yields

$\underline{\text { Order } \varepsilon^{0}}$

$$
\cos \left(\sigma T_{1}+\frac{T_{0} \omega_{n}}{2}\right) \zeta_{0}+\omega_{n}^{2} a_{10}+a_{10}^{(2,0)}=0
$$

\section{$\underline{\text { Order } \varepsilon^{1}}$}

$$
\begin{array}{r}
a_{10}^{2} \chi_{2}+a_{10}^{3} \chi_{3}+a_{10}^{4} \chi_{4}+a_{10}^{5} \chi_{5}+a_{10}^{6} \chi_{6}+a_{10}^{7} \chi_{7} \\
+a_{10} c \mu_{1} a_{10}{ }^{(1,0)}+a_{10}^{2} c \mu_{2} a_{10}{ }^{(1,0)}+a_{10}^{3} c \mu_{3} a_{10}{ }^{(1,0)} \\
+\cos \left(\sigma T_{1}+T_{0} \omega_{n}\right) \zeta_{3}+\cos \left(\sigma T_{1}+T_{0} \omega_{n}\right) a_{10} \zeta_{4} \\
+a_{10}^{4} c \mu_{4} a_{10}{ }^{(1,0)}+a_{10} \mu_{1} a_{10}{ }^{(2,0)}+a_{10}^{2} \mu_{2} a_{10}{ }^{(2,0)} \\
+a_{11} \omega_{n}^{2}+\cos a_{10}^{2} \zeta_{5}\left(\sigma T_{1}+T_{0} \omega_{n}\right)+c a_{10}{ }^{(1,0)} \\
+2 a_{10}{ }^{(1,1)}+a_{10}^{3} \mu_{3} a_{10}^{(2,0)}+a_{10}^{4} \mu_{4} a_{10}{ }^{(2,0)} \\
+a_{11}{ }^{(2,0)}+\cos \left(\sigma T_{1}+\frac{T_{0} \omega_{n}}{2}\right) a_{10} \zeta_{1} \\
+\cos \left(\sigma T_{1}+\frac{T_{0} \omega_{n}}{2}\right) a_{10}^{2} \zeta_{2}=0
\end{array}
$$


where $a_{i}^{(j, k)}=\frac{\partial^{k}}{\partial^{k} T_{1}^{k}}\left(\frac{\partial^{j}}{\partial^{j} T_{0}^{j}}\right)$.

The general solution of Eq. (23) can be written as

$$
a_{01}=A \cos \left(\omega_{n} T_{0}+\Phi\right)-\frac{4 \zeta_{0}}{3 \omega_{n}^{2}} \cos \left(\frac{\omega_{n} T_{0}}{2}+\sigma T_{1}\right)
$$

Equation (25) is then substituted in Eqns. (24) and the trigonometric functions are expanded. The elimination of the secular terms yields two first order non-linear ordinarydifferential equations which describe the amplitude and phase modulation of the response and permit a stability analysis

$$
\begin{aligned}
& \dot{A}=f_{1}(\varepsilon, A, \beta)+O\left(\varepsilon^{2}\right) \\
& \dot{\beta}=f_{2}(\varepsilon, A, \beta)+O\left(\varepsilon^{2}\right)
\end{aligned}
$$

where $\beta=2 \sigma T_{1}-\Phi$. The steady-state motions occur when $\dot{A}=\dot{\beta}=0$, which corresponds to the fixed points of Eqns. (26) and (27). Thus, the frequency-response equation can be written in its parametric form with respect to the phase $\beta$ as a set of two equations

$$
\begin{aligned}
& A=K_{1}(\beta) \\
& \Omega=K_{2}(\beta)
\end{aligned}
$$

This analytic expression makes the model suitable for MEMS and NEMS designers as a fast and efficient tool for resonant sensor performances optimisation. The expressions of Eqns. (28) and (29) are quite large. For the sake of conciseness, they are not detailed here

\section{Results}

All the numerical computations leading to Eqns. (28) and (29) were carried out with the following set of parameters: $l=50 \mu m, b=0.5 \mu m, l_{a}=40 \mu m, g_{a}=500 \mathrm{~nm}$, $l_{d}=48 \mu \mathrm{m}, g_{d}=300 \mathrm{~nm}$, while $h, V_{a c}$ and $V_{d c}$ were used for parametric analysis.

As shown in Fig. 2, the analytical model enables the capture of all the nonlinear phenomena in the resonator dynamics and describes the competition between the hardening (presented by the mechanical nonlinearity) and the softening (presented by the electrostatic nonlinearity) behaviors. Remarkably, the mixed behavior [9] was not observed. Indeed, the superharmonic excitation filters out the effect of the fifth order nonlinear terms and the compensation of the nonlinearities by hysteresis suppression $[7,8]$ is possible at large amplitudes. The upper bound limit of such operation is obviously the pull-in detailed below, but first the bistability limit (critical amplitude) of the resonator under superharmonic excitation is investigated by comparison to the primary resonance case.

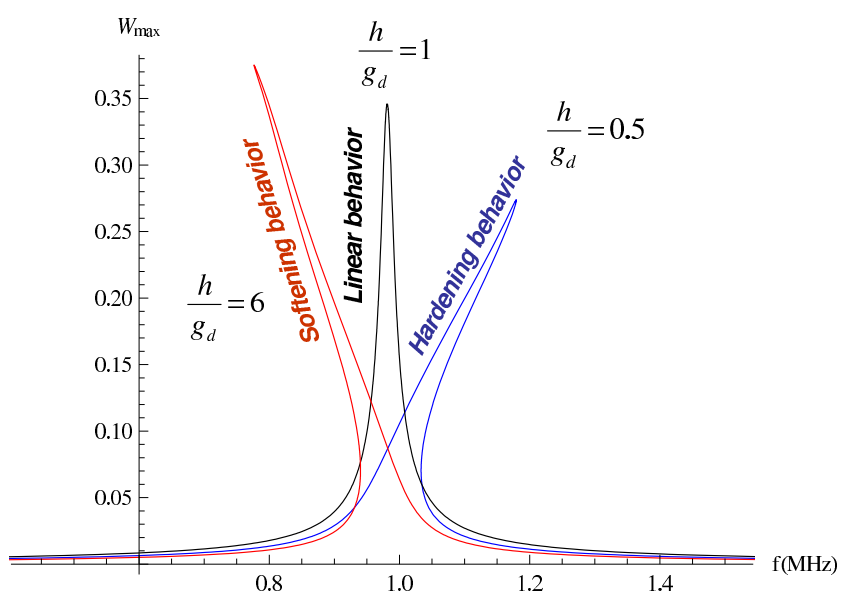

Fig. 2. Competition between hardening and softening behaviors for different values of the ratio $\frac{h}{g_{d}}$ ( $W_{\text {max }}$ is the normalized displacement at the middle of the beam)

\subsection{Critical amplitude}

As shown in Fig. 3, the critical amplitude is the oscillation amplitude $A_{c}$ above which bistability occurs. Thus, $A_{c}$ is the transition amplitude from the linear to the nonlinear behavior. For the primary resonance of a clamped-clamped mi-

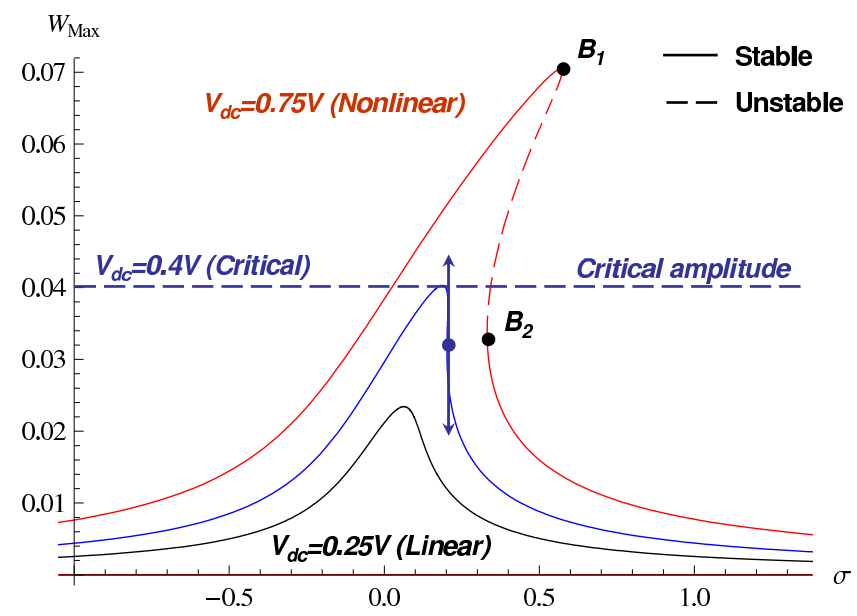

Fig. 3. Forced frequency responses of the typical resonator described in Fig. 1 for $h=0.5 \mu \mathrm{m}$ and $Q=5000$. $\sigma$ is the detuning parameter and $W_{\text {max }}$ is the displacement of the beam normalized by the gap $g_{d}$ at its middle point $\frac{l}{2} . B_{1}$ and $B_{2}$ are the two bifurcation points of a typical hardening behavior.

crobeam, $A_{c}$ is defined as the oscillation amplitude for which the equation $\frac{d \Omega}{d \beta}=0$ has a unique solution $\beta_{c}=\frac{2 \pi}{3}$ [27]. It was also shown analytically and experimentally $[7,28]$ that the critical amplitude for micromechanical clamped-clamped beam resonators under primary resonance is only determined by the beam thickness $h$ in the direction of vibration and the quality factor $Q$. The question is whether or not the critical 
amplitude of a NEMS resonator is invariant with respect to the excitation.

In order to explain how to deduce the mechanical critical amplitude from Eqns. (28) and (29), we assume the simplified case of neglected nonlinear electrostatic effects $\left(\frac{h}{g_{d}}<<1\right)$. Using Eq. (29), the derivative of the frequency $\Omega$ with respect to the phase $\beta$ is directly deduced as a function of several variables:

$$
\frac{d \Omega}{d \beta}=\Omega^{\prime}\left(\chi_{i}, \xi_{0}, \omega_{n}, \beta\right)
$$

One can search the condition for which the equation $\Omega^{\prime}\left(\chi_{i}, \xi_{0}, \omega_{n}, \beta\right)=0$ has a unique solution $\beta$. After some algebraic transformations, a relation between $\chi_{i}, \omega_{n}$ and $\xi_{0}$ has been identified and the critical amplitude of the electrostatic force $\xi_{0 c}$ is expressed as a function of the design and nonlinear parameters of the resonator. Explicitly, when the electrostatic forcing is $\xi_{0}=\xi_{0 c}, \Omega^{\prime}$ is null only at the critical phase $\beta_{c}=\frac{2 \pi}{3}$.

The critical amplitude is the value of Eq. (28) at the phase $\beta=\frac{\pi}{2}$ (for a critical behavior) multiplied by 1.588 which corresponds to the amplification coefficient of the first mode at the middle of the clamped-clamped beam $x=\frac{1}{2}$. Replacing $\xi_{0}$ by $\xi_{0 c}$ in the resulting equation and multiplying by the gap $g_{d}$ to obtain the dimensional value and using the expressions of the needed parameters from the appendix, yields:

$$
A_{c}=1.685 \frac{h}{\sqrt{Q}}
$$

It can be noted that this analytic expression for the critical amplitude is the same as in the case of primary resonance [7]. In order to validate this simple expression, some computations are carried out using Eqns. (28) and (29). The corresponding results are presented hereafter and the process leading to the dependency of $A_{c}$ on $h$ and $Q$ is explained graphically. The $A C$ voltage and the beam thickness are respectively fixed at $V_{a c}=0.6 \mathrm{~V}$ and $h=1 \mu \mathrm{m}$. Then, for three values of $D C$ voltage, $\frac{d \Omega}{d \beta}$ is calculated at the phase $\beta_{c}=\frac{2 \pi}{3}$ and plotted with respect to the quality factor $Q$ as shown in Fig. 4a. The critical quality factor $Q_{c}$ corresponds to the intersection between the curve $\frac{d \Omega\left(\frac{2 \pi}{3}\right)}{d \beta}[Q]$ and the $Q$-axis.

The critical amplitude is then the maximum amplitude of the frequency response curves plotted in $4 \mathrm{~b}$ for each of the three values of the critical quality factor $Q 1, Q 2$ and $Q 3$. At this point the dependency between the critical amplitude and the quality factor can be plotted, as seen in Fig. 4c. This permits to conclude that the critical amplitude $A_{c}$ decreases when the quality factor increases. Moreover, the identification of the coefficients $\operatorname{Coef}_{1}$ and Coef $_{2}$ such that $\log \left(A_{c}\right)=\operatorname{Coef}_{1}-\operatorname{Coef}_{2} \log (Q)$ leads to $\operatorname{Coef}_{1}=$ $\log \left(1.685 \frac{h}{g_{d}}\right)=0.448$ and $\operatorname{Coef}_{2}=-\frac{1}{2}$, which is in very good agreement with Eq. (31).

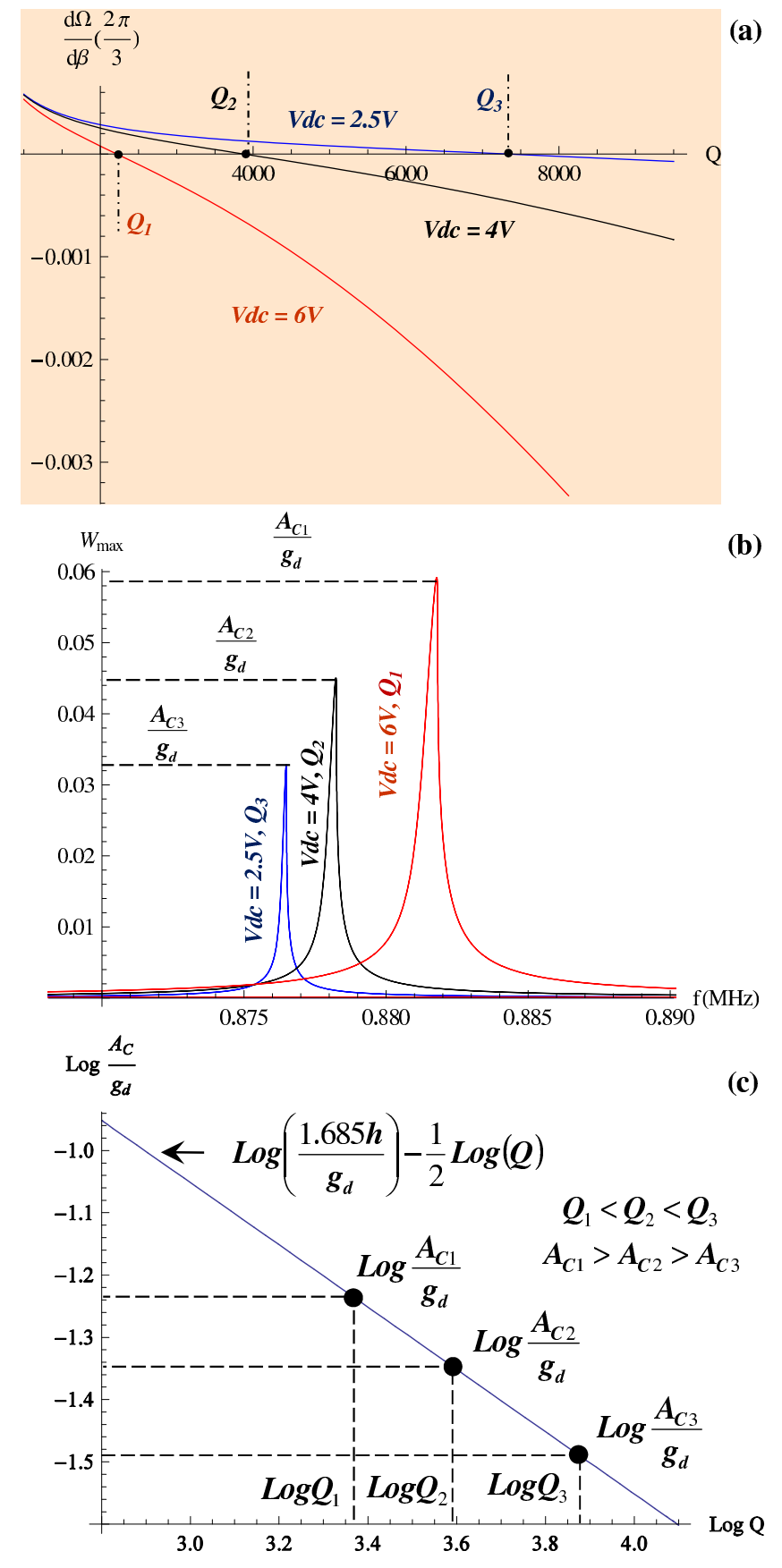

Fig. 4. (a): Critical quality factor determination for different $D C$ voltage $\left(h=500 \mathrm{~nm}\right.$ and $\left.V_{a c}=0.6 \mathrm{~V}\right)$. (b): Dependency of the critical amplitude on the quality factor (Here $A_{c}$ is the peak of $W_{\max }$ ). (c): Logarithmic plot of the critical amplitude with respect to the quality factor.

Figure 5a shows the variation of the critical thickness $h_{c}$ for different $D C$ polarizations $V_{d c}$. The fixed values of the $A C$ voltage and the quality factor $Q$ are $V_{a c}=0.6 \mathrm{~V}$ and $Q=4900$. The critical thickness $h_{c}$ is determined as the intersection with the horizontal axis in the same way as $Q_{c}$. These values are used to plot the frequency responses of Fig. $5 \mathrm{~b}$ and then Figure $5 \mathrm{c}$ is obtained, which clearly indicates the linear dependence of the critical amplitude $A_{c}$ on the thick- 
ness of the resonator. Again, these numerical results are in very good agreement with Eq. (31).
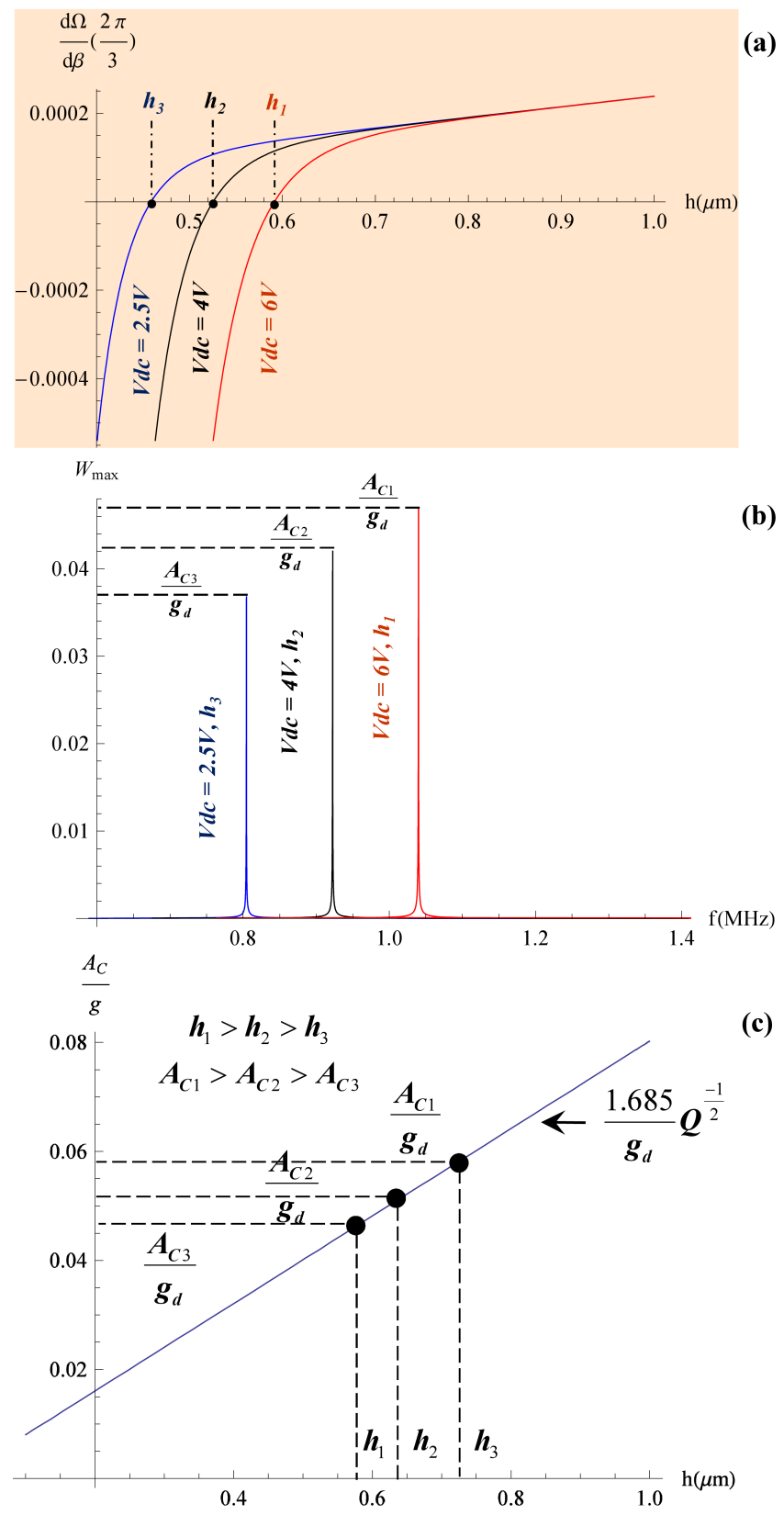

Fig. 5. (a): Critical thickness determination for different $D C$ voltage $\left(Q=4900\right.$ and $\left.V_{a c}=0.6 \mathrm{~V}\right)$. (b): Dependency of the critical amplitude on the thickness (Here $A_{c}$ is the peak of $W_{\max }$ ). (c): Plot of the critical amplitude with respect to the beam thickness.

\subsection{Pull-in retarding}

In order to make the detection technique easier for NEMS resonant sensors, it is convenient to drive the resonator at very large oscillations, i.e. beyond the critical amplitude $A_{c}$, which means in the nonlinear regime. However, at this vibration level, instabilities such as pull-in are likely to occur, which might cause the ruin of the sensor. Therefore, it is crucial for NEMS designers to predict the bistability limit (critical amplitude) as well as the upper bound amplitude not to be exceeded in order to avoid pull-in. In the following we not only propose a simple analytical expression for the pull-in amplitude, but also discuss a way to retard it.

\subsubsection{Pull-in amplitude}

The NEMS resonator must operate in the safe working range and away from instabilities. One of the main drawbacks with electrostatically actuated NEMS structures is the so-called pull-in instability, i.e., the collapse of the microbeam on the electrode, which has been investigated with both static [29] and dynamic [20,30] applied voltages.

The pull-in amplitude is the oscillation amplitude above which the resonator position becomes unstable and collapses. Several researchers predicted the presence of dynamic pullin in various beam-based systems at voltages lower than the static pull-in voltage [19, 20,30-32]. Fargas-Marques et al [33] used an energy-based analytical method for the dynamic pull-in prediction in electrostatic MEMS and provided experimental data. Ashhab et al [34] and Basso et al [35] have also investigated the problem of escape from potential well for atomic force microscopes (AFM), with the attractive force being the van der Waals forces.

Unlike these energy-based approaches, we investigate the pull-in phenomenon via the dynamic stability of a fixed point of the corresponding Poincaré map which is obviously a logical choice, given the developed reduced-order model and the resulting amplitude and phase modulation equations. More specifically, the initiation of the pull-in domain under superharmonic resonance has been located at an amplitude reaching an infinite slope at the phase $\beta_{p}=\frac{\pi}{2}$ which corresponds to a Floquet multiplier approaching unity $[7,19,20]$. For convenience, the lower bound of this domain is called "pull-in amplitude" since at this oscillation level which is beyond the critical amplitude, the beam can collapse.

Figure 6 a) shows several predicted frequency-response curves under superharmonic resonance. For the chosen design, the resonator operates in the nonlinear regime. As a result, the response amplitude are much greater than the normalized critical amplitude given by $\frac{A_{c}}{g_{d}}=0.1025$ which is the limit of the linear regime. For $V_{d c}=15 \mathrm{~V}$, the response curve exhibits a classical nonlinear hardening behavior with a maximum normalized amplitude $W_{\max }=0.26$. For $V_{d c}=20 \mathrm{~V}$, the response curve is highly nonlinear and the dynamic pullin occurs at $W_{\max } \simeq 0.35$ with a phase $\beta_{p}=\frac{\pi}{2}$ in Eqns. (28)(29). As shown on Figure $6 \mathrm{~b}$ ), the response curve also reaches an infinite slope at the pull-in amplitude. The resonator becomes highly unstable inside a narrow frequency zone surrounding the pull in amplitude. This frequency band corresponds to an escape zone where the kinetic energy of the nonlinear resonator exceeds the barrier of its potential well [38]. Inside this narrow zone, the nonlinear vibrations of the beam follow a pull-in attractor causing the damage of the NEMS sensor. This escape phenomenon is schematically represented by a bold point. It is worth noting that the present 
reduced-order model is capable of predicting the pull-in phenomenon with only one mode retained and a semi-analytical solving, in contrast with the purely numerical method used in [19].
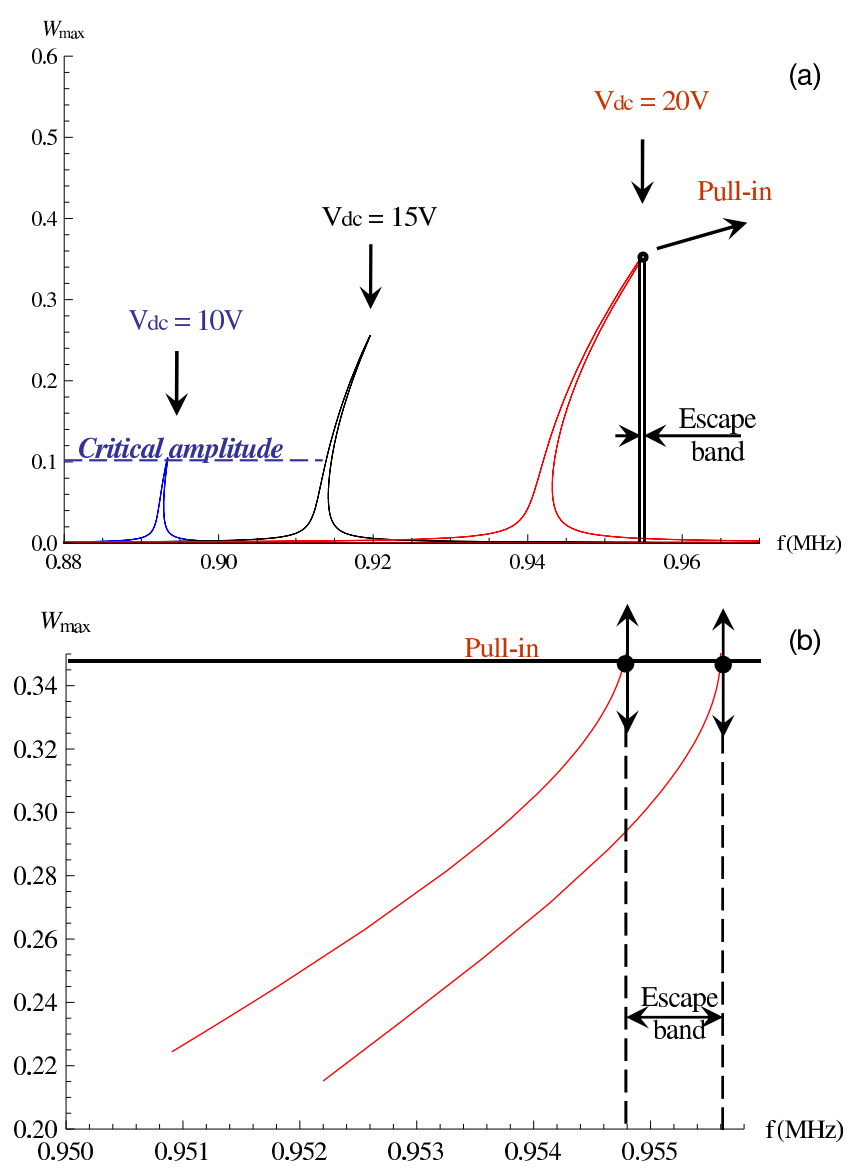

Fig. 6. (a): Predicted frequency curves for different $D C$ voltage $\left(Q=3000, h=1 \mu m\right.$ and $\left.V_{a c}=0.4 V\right)$. (b): Zoom on the escape band and the infinite slope at the phase $\beta=\frac{P i}{2}$ for $V_{d c}=20 \mathrm{~V}$.

\subsubsection{Pull-in control}

Methods have been employed so far to control pull-in phenomenon such the simple addition of a series capacitance [36] as well as the "voltage control algorithm". The latter has been applied by Chu and Pister [37]. It is based on controlling the voltage according to a feedback loop that exactly prevents the pull-in while taking into account the effect of nonlinearity for getting more exact solutions to this instability. Unlike these techniques, we propose a simple electrostatic mechanism based on the increase of the ratio between the applied $D C$ and $A C$ voltages.

Ideally, it is desirable to drive the resonator at large oscillations up to the gap $g_{d}$ in order to get the best signal to noise ratio as possible. However, this is hardly compatible with low dimensions devices and high quality factors according to Eq. (31). As a result, the critical amplitude is often very low and the operating range between the noise floor and the critical amplitude is very narrow, i.e. the device is prone to pull-in even at low displacements. As a consequence, resonators are generally designed to vibrate with amplitudes below a small fraction (less than a tenth) of the gap to guarantee the efficiency and no damage of the device. For further improvements of the sensors, MEMS and NEMS designers are interested in maximizing the detected signals in order to enable the electric characterization of electrostatically actuated nanoresonators with conventional measurement equipments. The control of the pull-in amplitude is thus of prime interest and the question is how to retard it.

In order to understand which parameters have an influence on the pull-in amplitude under superharmonic resonance, the $D C$ voltage is increased successively from $12 \mathrm{~V}$ to $25 \mathrm{~V}$ while lowering the $A C$ voltage from $0.5 \mathrm{~V}$ to $0.3 \mathrm{~V}$. Remarkably, the unavoidable pull-in escape frequency band is reached at larger oscillation amplitudes for low $V_{a c}$ and high $V_{d c}$ as shown in Fig. 7. The pull-in amplitude is increased from 0.25 up to 0.45 , which represents a $80 \%$ enhancement. This pull-in retarding mechanism with respect to the resonator displacement is purely electrostatic and it is caused by the amplification of the nonlinear electrostatic stiffness which balances the mechanical hardening nonlinearities and modifies the bifurcation topology of the expected dynamic response. Indeed, increasing the ratio $\frac{V_{d c}}{V_{a c}}$ while keeping a constant $V_{d c} . V_{a c}$ accelerates the spring softening effects and consequently, it enhances the stable oscillations of the resonator below the pull-in amplitude.

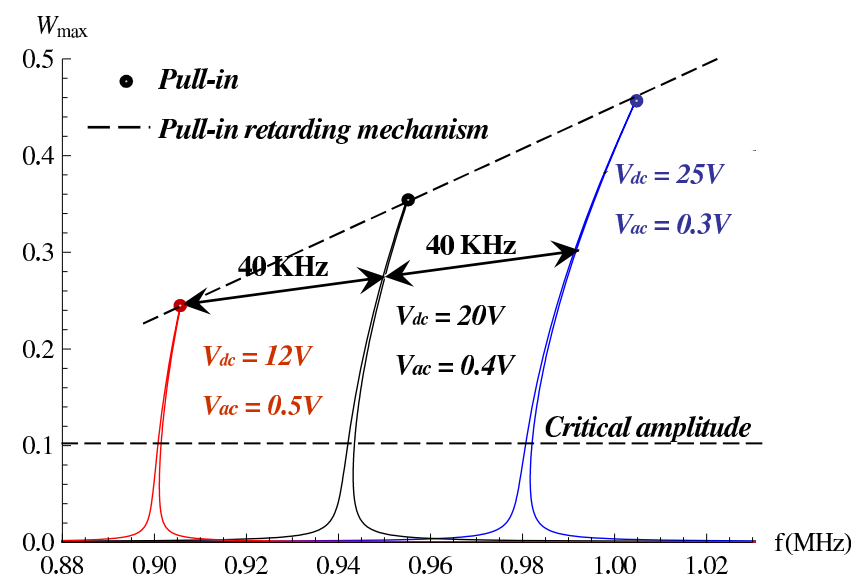

Fig. 7. Predicted frequency curves (up to pull-in) for different $A C$ and $D C$ polarizations $(Q=3000$ and $h=1 \mu \mathrm{m})$.

Figure 8 shows the variation of the resonant amplitude up to pull-in with respect to $A C$ voltage $V_{a c}$. For NEMS resonators under superharmonic resonance, it is possible to shift up the pull-in amplitude by applying a low $A C$ voltage. It is important to underline that this ability to control the pull-in amplitude with only one physical parameter (the driving $A C$ voltage) is not possible under primary resonance [7]. However, in order to compensate for the loss of performance, the 
resonator must be actuated with higher $D C$ polarizations.

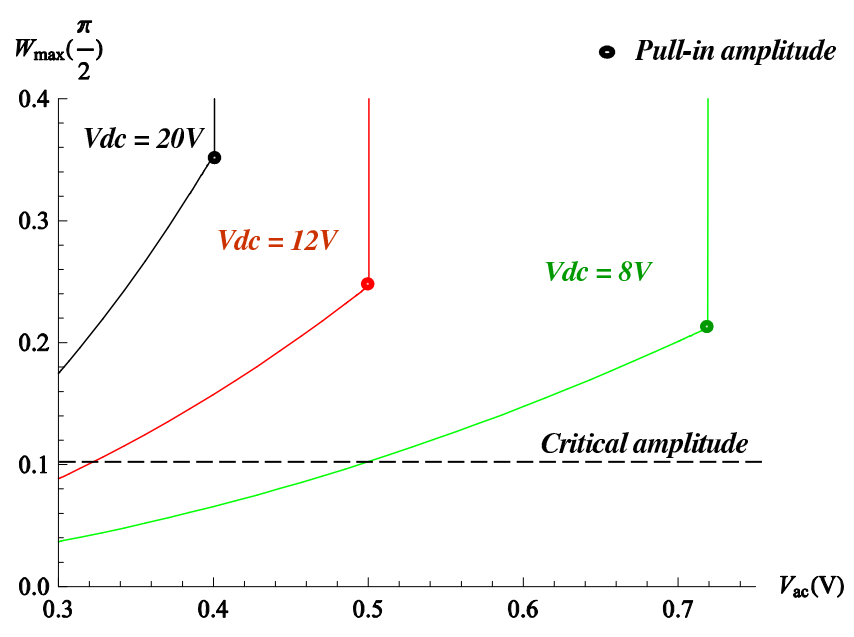

Fig. 8. Dependency of the pull-in amplitude on the $A C$ voltage.

\section{Conclusion}

A global approach to model and simulate the dynamics of NEMS resonators is presented. Particularly, superharmonic resonance excitation of order-two is investigated and results of analytical simulations are presented. They show that the superharmonic excitation filters out the mixed behavior [9] and the third order nonlinearity cancellation [5,7] is only limited by the pull-in. Moreover, compared to the primary resonance, it is demonstrated that the critical amplitude of the resonator does not change and keeps the same dependence on the quality factor $Q$ and the thickness $h$. Furthermore, the existence and the mechanism of the dynamic pull-in phenomenon under superharmonic excitation are analytically analyzed and close-form expressions for the pull-in amplitude as well as the pull-in voltage could be provided using the model. In practice, this analytical model constitutes a fast and efficient tool for NEMS designers for the evaluation of the appropriate $D C$ voltage and the amplitude and frequency of the $A C$ load in order to shift up or retard pullin while operating around the hysteresis suppression domain. Thus the resonator performances can be enhanced since driving it linearly at large amplitude could prevent most of noise mixing $[3,4]$.

\section{Acknowledgements}

The authors gratefully acknowledge financial support from the CEA LETI and I@L Carnot institutes (NEMS Project) and from the MNTEurop Project.

\section{Appendix}

$$
\begin{aligned}
& \omega_{n}^{2}=-2 V_{d c}^{2} \frac{C_{n 2} \alpha_{2}}{R_{g}} \int_{\frac{l-l_{d}}{2 l}}^{\frac{l+l_{d}}{2 l}} \phi_{1}^{2} d x \\
& -C_{n 1} \alpha_{2} \frac{2 V_{d c}^{2}+V_{a c}^{2}}{R_{g}^{2}} \int_{\frac{l-l_{a}}{2 l}}^{\frac{l+l_{a}}{2 l}} \phi_{1}^{2} d x \\
& +\lambda_{1}^{2}+12.3026 N \\
& \mu_{1}=2.65876 \frac{\left(1+R_{g}\right)}{R_{g}} \\
& \mu_{2}=1.8519 \frac{1+4 R_{g}+R_{g}^{2}}{R_{g}^{2}} \\
& \mu_{3}=5.30219 \frac{1+R_{g}}{R_{g}^{2}} \\
& \mu_{4}=-7.72721 \frac{1+R_{g}}{R_{g}^{2}} \\
& \chi_{2}=1.32938\left(2 \lambda_{1}^{2} \frac{\left(1+2 R_{g}\right)}{R_{g}}+\frac{C_{n 1} \alpha_{2} V_{a c}^{2}}{R_{g}^{2}}\right) \\
& +C_{n 2} \alpha_{2} \frac{\left(V_{d c}^{2}-2 V_{d c} V s+V s^{2}\right)}{R_{g}{ }^{2}} \int_{\frac{l-l_{d}}{2 l}}^{\frac{l+l_{d}}{2 l}} \phi_{1}^{3} d x \\
& -\frac{V_{d c}^{2} C_{n 1} \alpha_{2}}{R_{g}{ }^{2}} \int_{\frac{l-l_{a}}{2 l}}^{\frac{l+l_{a}}{2 l}} \phi_{1}^{3} d x \\
& +38.2811 N \frac{\left(1+2 R_{g}\right)}{R_{g}} \\
& \chi_{3}=28.2132 N \frac{1+4 R_{g}+R_{g}^{2}}{R_{g}^{2}}+151.354 \alpha_{1} \\
& +1.8519 \lambda_{1}^{2} \frac{1+4 R_{g}+R_{g}^{2}}{R_{g}^{2}} \\
& \chi_{4}=470.958 \alpha_{1} \frac{1+R_{g}}{R_{g}}+5.30219 \lambda_{1}^{2} \\
& +83.1444 N \frac{1+R_{g}}{R_{g}^{2}} \\
& \chi_{5}=3.8636 \frac{\lambda_{1}^{2}}{R_{g}^{2}}+61.6548 \frac{N}{R_{g}^{2}} \\
& +347.096 \alpha_{1}^{2}\left(1+4 R_{g}+R_{g}^{2}\right) \\
& \chi_{6}=1022.89 \alpha_{1} \frac{1+R_{g}}{R_{g}^{2}} \\
& \chi_{7}=758.515 \frac{\alpha_{1}}{R_{g}^{2}} \\
& \mathrm{v}=-\frac{1}{2}\left(C_{n 1} \alpha_{2}\left(V_{a c}^{2}+2 V_{d c}^{2}\right) \int_{\frac{l-l_{a}}{2 l}}^{\frac{l+l_{a}}{2 l}} \phi_{1} d x\right) \\
& +C_{n 2} R_{g}^{2} \alpha_{2}\left(V_{d c}^{2}+V s^{2}\right) \int_{\frac{l-l_{d}}{2 l}}^{\frac{l+l_{d}}{2 l}} \phi_{1} d x \\
& \zeta_{0}=4 V_{a c} V_{d c} C_{n 1} \alpha_{2} \int_{\frac{l-l_{a}}{2 l}}^{\frac{l+l_{a}}{2 l}} \phi_{1} d x
\end{aligned}
$$




$$
\begin{gathered}
\zeta_{1}=-4 V_{a c} V_{d c} C_{n 1} \alpha_{2} \int_{\frac{l-l_{a}}{2 l}}^{\frac{l+l_{a}}{2 l}} \phi_{1}^{2} d x \\
\zeta_{2}=2 V_{a c} V_{d c} C_{n 1} \alpha_{2} \int_{\frac{l-l_{a}}{2 l}}^{\frac{l+l_{a}}{2 l}} \phi_{1}^{3} d x
\end{gathered}
$$

\section{References}

[1] Feng, X. L., 2007. "Phase noise and frequency stability of very-high frequency silicon nanowire nanomechanical resonators". In 14th International Conference on Solid-State Sensors, Actuators and Microsystems, pp. 327-30.

[2] Robins, W. P., 1984. Phase Noise in Signal Sources. Institution of Engineering and Technology.

[3] Roessig, T. A., Howe, R. T., and Pisano, A. P., 1997. "Nonlinear mixing in surface-micromachined tuning fork oscillators". In Frequency Control Symposium, 1997., Proceedings of the 1997 IEEE International, pp. 778-782.

[4] Kaajakari, V., Koskinen, J. K., and Mattila, T., 2005. "Phase noise in capacitively coupled micromechanical oscillators". Ultrasonics, Ferroelectrics and Frequency Control, IEEE Transactions on, 52(12), Dec., pp. 2322-2331.

[5] Kacem, N., Hentz, S., Fontaine, H., Nguyen, V., Robert, P., Legrand, B., and Buchaillot, L., 2008. "From mems to nems: Modelling and characterization of the non linear dynamics of resonators, a way to enhance the dynamic range". In Int. Conf. Nanotech, (Boston : Massachusetts, U.S.A).

[6] Postma, H. W. C., Kozinsky, I., Husain, A., and Roukes, M. L., 2005. "Dynamic range of nanotubeand nanowire-based electromechanical systems". Applied Physics Letters, 86(22), p. 223105.

[7] Kacem, N., Hentz, S., Pinto, D., Reig, B., and Nguyen, V., 2009. "Nonlinear dynamics of nanomechanical beam resonators: improving the performance of nemsbased sensors". Nanotechnology, 20(27), p. 275501.

[8] Kacem, N., Arcamone, J., Perez-Murano, F., and Hentz, S., 2010. "Dynamic range enhancement of nonlinear nanomechanical resonant cantilevers for highly sensitive nems gas/mass sensor applications". Journal of Micromechanics and Microengineering, 20(4), p. 045023.

[9] Kacem, N., and Hentz, S., 2009. "Bifurcation topology tuning of a mixed behavior in nonlinear micromechanical resonators". Applied Physics Letters, 95(18), p. 183104.

[10] Kacem, N., Baguet, S., Hentz, S., and Dufour, R., 2010. "Nonlinear phenomena in nanomechanical resonators: mechanical behaviors and physical limitations". Mécanique \& Industries, 11(6), pp. 521-529.

[11] Jin, Z., and Wang, Y., 1998. "Electrostatic resonator with second superharmonic resonance". Sensors and Actuators A: Physical, 64(3), pp. 273 - 279.

[12] Turner, K. L., Miller, S. A., Hartwell, P. G., MacDonald, N. C., Strogatz, H. S., and Adams, S. G., 1998.
"Five parametric resonances in a microelectromechanical system". Nature, 396, pp. 149-152.

[13] Rugar, D., and Grütter, P., 1991. "Mechanical parametric amplification and thermomechanical noise squeezing". Phys. Rev. Lett., 67(6), Aug, pp. 699-702.

[14] Carr, D. W., Evoy, S., Sekaric, L., Craighead, H. G., and Parpia, J. M., 2000. "Parametric amplification in a torsional microresonator". Applied Physics Letters, 77(10), pp. 1545-1547.

[15] Carr, D. W., Evoy, S., Sekaric, L., Craighead, H. G., and Parpia, J. M., 1999. "Measurement of mechanical resonance and losses in nanometer scale silicon wires". Applied Physics Letters, 75(7), pp. 920-922.

[16] Younis, M., and Nayfeh, A. H., 2003. "A study of the nonlinear response of a resonant microbeam to an electric actuation". Nonlinear Dynamics, 31(1), pp. 91117.

[17] Abdel-Rahman, E. M., and Nayfeh, A. H., 2003. "Secondary resonances of electrically actuated resonant microsensors". Journal of Micromechanics and Microengineering, 13(3), pp. 491-501.

[18] Younis, M. I., Abdel-Rahman, E. M., and Nayfeh, A. H., 2004. "Global dynamics of mems resonators under superharmonic excitation". In the International Conference on MEMS, NANO, and Smart Systems, Banff, Canada (ICMENS), p. 694699.

[19] Nayfeh, A. H., and Younis, M. I., 2005. "Dynamics of mems resonators under superharmonic and subharmonic excitations". Journal of Micromechanics and Microengineering, 15(10), pp. 1840-1847.

[20] Nayfeh, A. H., Younis, M. I., and Abdel-Rahman, E. M., 2007. "Dynamic pull-in phenomenon in mems resonators". Nonlinear Dynamics, 48, pp. 153-163.

[21] Landau, L. D., and Lifshitz, E. M., 1986. Theory of Elasticity. Butterworth-Heinemann.

[22] Nishiyama, H., and Nakamura, M., 1990. "Capacitance of a strip capacitor". Components, Hybrids, and Manufacturing Technology, IEEE Transactions on, 13(2), Jun, pp. 417-423.

[23] Touzé, C., and Thomas, O., 2006. "Non-linear behaviour of free-edge shallow spherical shells: Effect of the geometry". International Journal of Non-Linear Mechanics, 41(5), pp. 678-692.

[24] Kacem, N., Baguet, S., Hentz, S., and Dufour, R., 2011. "Computational and quasi-analytical models for nonlinear vibrations of resonant mems and nems sensors". International Journal of Non-Linear Mechanics, 46(3), pp. 532-542.

[25] Belhaq, M., and Fahsi, A., 2008. "2:1 and 1:1 frequency-locking in fast excited van der polmathieuduffing oscillator". Nonlinear Dynamics, 53, pp. 139-152.

[26] Nayfeh, A. H., 1981. Introduction to Perturbation Techniques. Wiley.

[27] Yurke, B., Greywall, D. S., Pargellis, A. N., and Busch, P. A., 1995. "Theory of amplifier-noise evasion in an oscillator employing a nonlinear resonator". Phys. Rev. A, 51(5), May, pp. 4211-4229. 
[28] Shao, L. C., Palaniapan, M., and Tan, W. W., 2008. "The nonlinearity cancellation phenomenon in micromechanical resonators". Journal of Micromechanics and Microengineering, 18(6), p. 065014.

[29] Younis, M. I., Abdel-Rahman, E. M., and Nayfeh, A., 2003. "A reduced-order model for electrically actuated microbeam-based mems". Microelectromechanical Systems, Journal of, 12(5), oct., pp. 672-680.

[30] Krylov, S., and Maimon, R., 2004. "Pull-in dynamics of an elastic beam actuated by continuously distributed electrostatic force". Journal of Vibration and Acoustics, 126(3), pp. 332-342.

[31] De, S. K., and Aluru, N. R., 2004. "Full-lagrangian schemes for dynamic analysis of electrostatic mems". Microelectromechanical Systems, Journal of, 13(5), oct., pp. 737-758.

[32] Elata, D., and Bamberger, H., 2006. "On the dynamic pull-in of electrostatic actuators with multiple degrees of freedom and multiple voltage sources". Microelectromechanical Systems, Journal of, 15(1), feb., pp. 131-140.

[33] Fargas-Marques, A., Casals-Terre, J., and Shkel, A. M., 2007. "Resonant pull-in condition in parallel-plate electrostatic actuators". Microelectromechanical Systems, Journal of, 16(5), oct., pp. 1044-1053.

[34] Ashhab, M., Salapaka, M. V., Dahleh, M., , and Mezic, I., November 1999. "Melnikov-based dynamical analysis of microcantilevers in scanning probe microscopy". Nonlinear Dynamics, 20, pp. 197-220(24).

[35] Basso, M., Giarre, L., Dahleh, M., and Mezic, I., 2000. "Complex dynamics in a harmonically excited lennardjones oscillator: Microcantilever-sample interaction in scanning probe microscopes". Journal of Dynamic Systems, Measurement, and Control, 122(1), pp. 240-245.

[36] Seeger, J. I., and Crary, S. B., 1997. "Stabilization of electrostatically actuated mechanical devices". In Solid State Sensors and Actuators, 1997, Vol. 2, pp. 1133 1136 vol.2.

[37] Chu, P. B., and Pister, S. J., 1994. "Analysis of closedloop control of parallel-plate electrostatic microgrippers". In Robotics and Automation, 1994. Proceedings., 1994 IEEE International Conference on, pp. 820 -825 vol.1.

[38] Gottwald, J. A., Virgin, L. N., and Dowell, E. H., 1995. "Routes to escape from an energy well". Journal of Sound and Vibration, 187(1), pp. 133 - 144.

\section{List of Figures}

1 Schema of an electrically actuated microbeam

2 Competition between hardening and softening behaviors for different values of the ratio $\frac{h}{g_{d}}\left(W_{\max }\right.$ is the normalized displacement at the middle of the beam) . . . . . . . . . .

3 Forced frequency responses of the typical resonator described in Fig. 1 for $h=0.5 \mu \mathrm{m}$ and $Q=5000$. $\sigma$ is the detuning parameter and $W_{\max }$ is the displacement of the beam normalized by the gap $g_{d}$ at its middle point $\frac{l}{2}$. $B_{1}$ and $B_{2}$ are the two bifurcation points of a typical hardening behavior. . . . . . . .

4 (a): Critical quality factor determination for different $D C$ voltage $\left(h=500 \mathrm{~nm}\right.$ and $V_{a c}=$ $0.6 \mathrm{~V})$. (b): Dependency of the critical amplitude on the quality factor (Here $A_{c}$ is the peak of $\left.W_{\max }\right)$. (c): Logarithmic plot of the critical amplitude with respect to the quality factor. . . . . . . . . . . .

5 (a): Critical thickness determination for different $D C$ voltage $\left(Q=4900\right.$ and $V_{a c}=$ $0.6 V)$. (b): Dependency of the critical amplitude on the thickness (Here $A_{c}$ is the peak of $\left.W_{\max }\right)$. (c): Plot of the critical amplitude with respect to the beam thickness. . . . . . .

6 (a): Predicted frequency curves for different $D C$ voltage $\left(Q=3000, h=1 \mu m\right.$ and $V_{a c}=$ $0.4 V)$. (b): Zoom on the escape band and the infinite slope at the phase $\beta=\frac{P i}{2}$ for $V_{d c}=20 \mathrm{~V} . \quad 8$

7 Predicted frequency curves (up to pull-in) for different $A C$ and $D C$ polarizations $(Q=3000$ and $h=1 \mu m) \ldots \ldots \ldots$. . . . . . . 8

8 Dependency of the pull-in amplitude on the $A C$ voltage. . . . . . . . . . . . 9 